\title{
A Power Allocation Scheme Using Updated SLNR Value Based on Perturbation Theory*
}

\author{
Wenwen $\mathrm{Cao}^{1}$, Zi Teng ${ }^{1,2}$, Jun $\mathrm{Wu}^{1}$ \\ ${ }^{1}$ College of Electronics and Information Engineering, Tongji University, Shanghai, 201804, China \\ ${ }^{2}$ School of Mathematics, Physics \& Information Engineering, Jiaxing University, Jiaxing, Zhejiang, China. \\ E-mail: \{1131660, 2011 tengz, wujun $\} @$ tongji.edu.cn
}

Received June, 2013

\begin{abstract}
The performance of downlink multiple-input multiple-output (MIMO) cellular networks is limited by co-channel interference (CCI). In this paper, we propose a linear precoding scheme based on signal-to-leakage-and-noise ratio (SLNR) criteria which can reduce the CCI significantly. Since each user's SLNR value is corresponding to the largest eigenvalue of the generalized matrix which indicates the channel quality that we propose a scheme to do a dynamic power allocation as an auxiliary way to improve SLNR precoding scheme. We use the perturbation theory to update each user's SLNR value each time step in time-varying channels rather than directly decompose the channel matrix so as to reduce the amount of calculation. The simulation results show that the proposed scheme offers about $0.3 \mathrm{bps} / \mathrm{Hz}$ additional capacity gain and $0.5 \mathrm{~dB}$ BER gain over conventional SLNR precoding method with lower computational complexity. And it also obtains about $0.5 \mathrm{bps} / \mathrm{Hz}$ additional capacity gain and $1 \mathrm{~dB}$ BER gain compared to the scheme only update the preceding vectors.
\end{abstract}

Keywords: Updated SLNR Value; Perturbation Theory; Power Allocation

\section{Introduction}

In downlink multi-user MIMO system, because of the limited frequency resources, a base station (BS) has to communicate with several co-channel user Equipments (UEs) to achieve high system capacity. This way of transmission inevitably causes co-channel interference. In general, there are non-linear and linear MU-MIMO precoding schemes to solve this problem. Due to the high complexity, the nonlinear schemes $[1,2]$ are seldom used in practical. Only linear MU-MIMO precoding schemes are applied in 3GPP long-term evolution (LTE) [3]. The conventional linear schemes such as ZF (Zero- Forcing) [4-6], MMSE (Minimum Mean-Square Error) [4,6] and $\mathrm{BD}$ (block diagonalization) [7] can eliminate or reduce the interference among users and data streams. But all these solutions have the drawbacks that the number of base station antennas must be equal or greater than the data streams of all users. In [8], the SINR(Signal to Interference plus Noise Ratio) precoding has been proposed which is desirable to maximize the average SINR for designing a robust precoder connected to maximize the sum rate. But the algorithm contains iterative part which increases the complexity of the realization. How-

\footnotetext{
*This work is financially supported by NSFC General Program under contract No.61173041.
}

ever, the expression of SINR is coupled for each user that the solution of precoder vectors is difficult.

In $[9,10]$, SLNR (signal to leakage and noise ratio) has been developed to suppress the CCI. This scheme takes a comprehensive consideration of the useful signal, interference signal and channel noise. For each user, the transmitter only needs to work out a generalized eigenvalue to obtain the optimal precoding matrix. There is no constraint on the system configuration in terms of the number of transmit and receive antennas. So the SLNR precoding achieves a good tradeoff of algorithm complexity and system performance. In practical wireless communication system, because of the infraction and scattering, there exists time-delay and multipath-fading. In [11], the author has mentioned to employ a perturbation theory to update formula of precoding vector design as an approximate solution with tolerable small performance loss under time-varying channels. This method gives us an inspiration that since each user's SLNR value is corresponding to the largest eigenvalue of the generalized vector, we can use the perturbation theory to update each user's SLNR value as well as the generalized eigenvector corresponding to the slowly varying channel information.

In the most of existing precoding methods, it is always 
assumed that the transmitter distributes equal power to each user. But in the real wireless communication system, the users usually have different channel states. In $[12,13]$, the multi-user iterative water filling algorithm has been proposed. Although it achieves a good performance knowing the perfect CSI, its high complexity restricts the method to be put into practice. According to this classic power allocation approach, it is very important to note here that we tend to allocate more power to users in good channel condition in order to obtain better system performance. Thus, if the transmit power is allocated according to the accuracy CSI, some gains may be achieved due to the effective power allocation. In conventional SLNR precoding scheme, the SLNR value seems useless, but in our proposed scheme, we use each user's updated SLNR value as the refernece for power allocation to improve the system performance.

Notation: Throughout this paper, matrices are denoted by boldface symbols. $(\cdot)^{H}$ denotes the conjugate transpose, $\|\cdot\|_{F}$ represents the Frobenius norm, $I_{N}$ is the $N \times N$ identity matrix. $\mathbb{C}^{M \times N}$ represents the set of $M \times N$ matrices in complex field. Besides, $\dot{x}$ means $\mathrm{x}$ is differentiable.

The paper is organized as follows. The system model, previous works done on SLNR precoding, and the solution of updated eigenvalue based on perturbation theory are introduced in Section II. We propose a new power allocation scheme using the updated SLNR value in Section III. Numerical results and conclusion are provided in Section IV and Section V.

\section{System Model}

We consider a downlink MU-MIMO system in Figure 1, where there is a base station communicating with $K$ users simultaneously over the same time-frequency resource. The base station has $N$ transmit antennas and each user is equipped with $M_{k}$ antenna. We employ a linear precoding matrix at the transmitter, so the transmit data symbol vector $\mathbf{x} \in \mathbb{C}^{N \times 1}$ can be presented as

$$
\mathbf{X}=\sum_{k=1}^{\mathrm{K}} \mathbf{w}_{k} \mathbf{S}_{k}
$$
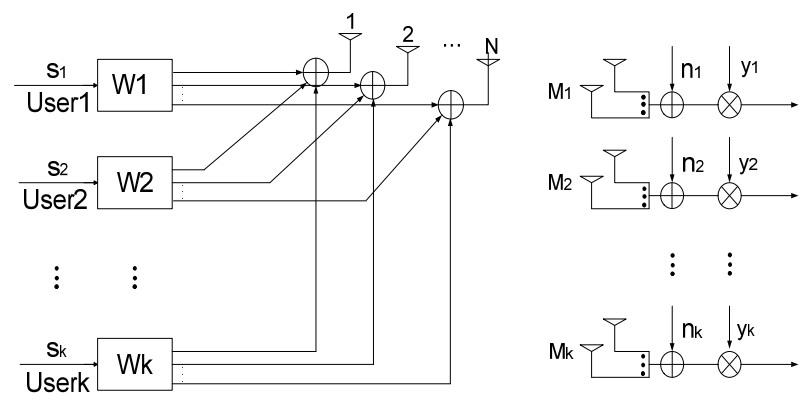

Figure 1. A block diagram of downlink MU-MIMO system. where $\mathbf{s}_{k} \in \mathbb{C}^{L \times 1}$ denotes the transmitted data for $k$-th user. $L$ is the number of data streams supported for user $k$ and it is assumed equal for all the users for simplicity. Here $\mathrm{L} \leq \mathrm{M}_{k}$ and $\mathrm{N} \geq \mathrm{LM}_{k}$. The scalar data $\mathbf{s}_{k}$ is multiplied by a $\mathrm{N} \times 1$ precoding vector before being transmitted over the channel. We can set $E \mathbf{x}^{2} \leq p_{0}$ as the transmit power constraint. And $p_{0}$ is the total transmit power of downlink.

For a given user, the received signal vector $\mathrm{y}_{k}$ at the $k$-th receiver is

$$
\mathbf{y}_{k}=\mathbf{H}_{k} \mathbf{x}_{k}+\mathbf{H}_{k} \sum_{i=1, i \neq k}^{\mathrm{K}} \mathbf{x}_{i}+\mathbf{n}_{k}
$$

where the received signal vector of user $k$ is denoted as $\mathbf{y}_{k} \in \mathbb{C}^{M_{k} \times 1}$. MIMO channel for the $k$-th user is $\mathbf{H}_{k} \in$ $\mathbb{C}^{M_{k} \times N}$. It is modeled as independent and identically distributed complex Gaussian variables with zero-mean and unit-variance. The noise $\mathbf{n}_{k} \in \mathbb{C}^{M_{k} \times 1}$ is independent complex Gaussian distributed, i.e $\mathbf{n}_{\mathrm{k}} \sim C N\left(0, \sigma_{k}^{2} \mathbf{I}_{M_{k}}\right)$.

\subsection{Signal to Leakage and Noise Ratio}

The SLNR precoding design makes a balance between eliminating the co-channel interference and the noise. The basic concept in a SLNR system is that it maximizes the strength of the desired signal relative to the noise and total interference caused to the other users [9]. This approach is discussed below.

We can use the linear SLNR precoder in [9] as an optimization metric. Since each user's precoding vector can be optimized, the optimization problem turns into a completely decoupled one. So we can obtain a closed-form solution. Besides, the SLNR precoding scheme does not need the constraint on the system configuration in terms of the number of transmit and receive antennas.

From the definition in [9], the $k$-th user's SLNR is

$$
\operatorname{SLNR}_{k}=\frac{\mathbf{H}_{k} \mathbf{w}_{k}^{2}}{\mathrm{M}_{k} \sigma^{2}+\sum_{i=1, i \neq \mathbf{F}}^{\mathrm{K}} \mathbf{H}_{i} \mathbf{w}_{k}^{2}{ }_{\mathrm{F}}^{2}} 1 \leq i \leq \mathrm{K}
$$

The SLNR precoding scheme deals with the total interfering power that user $i$ causes on all other users. The robust precoding vectors can be achieved by solving the following optimization problem. The precoder design aims at maximizing the SLNR can be formulated as

$$
\mathbf{w}_{k}^{o p t}=\arg \max _{\mathbf{w}_{k}} S L N R_{k} \quad 1 \leq k \leq \mathrm{K}
$$

Using the criterion of max each user's own SLNR value can decouple the precoding vector $\left\{\mathbf{w}_{k}\right\}$ in the objective function of (3), in [9], the optimum solution is given by

$$
\mathbf{w}_{k} \propto \text { eigvector }\left(\left(\mathrm{M}_{k} \sigma_{k}^{2}+\mathbf{H}_{k}^{\mathrm{H}} \mathbf{H}_{k}\right)^{-1} \mathbf{H}_{k}^{\mathrm{H}} \mathbf{H}_{k}\right)
$$

By generalized eigenvalue decomposition, there exists an invertible matrix $\mathbf{T}_{k} \in \mathbb{C}^{N \times N}$, 


$$
\left(\mathbf{M}_{k} \sigma_{k}^{2}+\mathbf{H}_{k}^{H} \mathbf{H}_{k}\right)^{-1} \mathbf{H}_{k}^{H} \mathbf{H}_{k} \mathbf{T}_{k}=\Lambda_{k}=\operatorname{diag}\left(\lambda_{1}, \cdots, \lambda_{N}\right)
$$

The maximum SLNR value is given by

$$
\operatorname{SLNR}_{k}^{\max }=\max \left(\lambda_{1}, \cdots, \lambda_{N}\right)
$$

In the conventional SLNR precoding approach, it is assumed that equal power is allocated to each user. As the SLNR value is available to the transmitter and it can indicate the channel quality of each user, we should take advantage of the knowledge of SLNR value which may bring further improvement to the system performance.

\subsection{Calculate Updated SLNR Value Using Perturbation theory}

We assume that the time-varying channel is slow fading which suits the practical wireless communication system. Actually, at two consecutive time steps, the transmit channels are not independent that we view the current channel as a slightly updated version of the previous one [14]. If we do the eigen-decomposition every time step, it will be a burdensome to calculation. So we should make full use of the time dependency of the channels between two consecutive time steps. Here the perturbation theory will be applied in calculating the current updated version of eigenvalues and eigenvectors.

The perturbation theory [15] considers the effect of a small disturbance in the equation and finds an approximate solution to a problem which cannot be solved exactly. It often provides a better approximate answer to what the real solution should be. Our approach is to apply matrix perturbation theory to the SLNR linear precoding where eigenvalues and eigenvectors are computed at every time step not using the matrix decomposition which reduces the complexity of computation.

Let $\mathbf{A}, \mathbf{B} \in \mathbb{C}^{\mathrm{m} \times \mathrm{n}}$, the definition of the generalized eigen equation is $\mathbf{A} \mathbf{x}_{i}=\lambda_{i} \mathbf{B} \mathbf{x}_{i}$. Where $\mathbf{x}_{i} \in \mathbb{C}^{\mathrm{n}}, \mathbf{x}_{i}$ is called a generalized eigenvector and $\lambda_{i}$ is called a generalized eigenvalue. When the equation is perturbed, we bring a parameter $\varepsilon$ to illustrate the change of the equation. By the definition, we know that

$$
(\mathbf{A}+\varepsilon \tilde{\mathbf{A}}) \mathbf{x}_{i}(\varepsilon)=\lambda_{i}(\varepsilon)(\mathbf{B}+\varepsilon \tilde{\mathbf{B}}) \mathbf{x}_{i}
$$

where $\varepsilon \ll 1$. Let $\left\{\lambda_{i}(\varepsilon)\right\}$ and $\left\{\mathrm{x}_{i}(\varepsilon)\right\}$ be the set of generalized eigenvalues and eigenvectors. The problem is to find the nontrivial solutions of equation (8).

When we solve the equation $(8), \lambda_{i}(\varepsilon)$ and $\mathrm{x}_{i}(\varepsilon)$ are differentiable about $\varepsilon$. Set $\varepsilon=0$ in the equation and the initial conditions to derive an initial value problem which determines the unperturbed solution $\lambda_{i}(0)$. The equation (8) can be transformed to be

$$
\begin{aligned}
& \mathbf{A} \dot{\mathbf{x}}_{i}(0)+\tilde{\mathbf{A}} \mathbf{x}_{i}(0) \\
= & \lambda_{i}(0) \mathbf{B} \dot{\mathbf{x}}_{i}(0)+\lambda_{i}(0) \tilde{\mathbf{B}} \mathbf{x}_{i}(0)+\dot{\lambda}_{i}(0) \mathbf{B} \mathbf{x}_{i}(0)
\end{aligned}
$$

$\lambda_{i}(\varepsilon)$ and $\mathrm{x}_{i}(\varepsilon)$ can be expressed in the form of Taylor expansion.

$$
\begin{aligned}
& \lambda_{i}(\varepsilon)=\lambda_{i}(0)+\varepsilon \dot{\lambda}_{i}(0)+\mathrm{O}\left(\varepsilon^{2}\right) \\
& \mathrm{x}_{i}(\varepsilon)=\mathrm{x}_{i}(0)+\varepsilon \dot{\mathrm{x}}_{i}(0)+\mathrm{O}\left(\varepsilon^{2}\right)
\end{aligned}
$$

All of the other terms in the linear equation are of order $\mathrm{O}\left(\varepsilon^{2}\right)$. By substituting the expansion (10) and (11) into the differential equation (9), we obtain solutions of the eigenvalues and eigenvectors to first order

$$
\begin{gathered}
\lambda_{i}(\varepsilon) \approx \lambda_{i}+\varepsilon \mathrm{x}_{i}^{\mathrm{H}}\left(\tilde{\mathbf{A}}-\lambda_{i} \tilde{\mathbf{B}}\right) \mathrm{x}_{i} \\
\mathrm{x}_{i}(\varepsilon) \approx \mathrm{x}_{i}\left(1-\frac{\varepsilon}{2} \mathrm{x}_{i}^{\mathrm{H}} \tilde{\mathbf{B}} \mathrm{x}_{i}\right)+\sum_{j=1, j \neq i}^{\mathrm{N}} \frac{\varepsilon \mathrm{x}_{j}^{\mathrm{H}}\left(\tilde{\mathbf{A}}-\lambda_{i} \tilde{\mathbf{B}}\right) \mathrm{x}_{i}}{\lambda_{i}-\lambda_{j}} \mathrm{x}_{j}
\end{gathered}
$$

In (12), the updated eigenvalues are formed by the unperturbed solution $\lambda_{i}$ and the first-order perturbation correction $\Delta \lambda_{i}(\varepsilon)=\varepsilon \mathrm{X}_{\mathrm{i}}^{\mathrm{H}}\left(\tilde{\mathbf{A}}-\lambda_{i} \tilde{\mathbf{B}}\right) \mathrm{X}_{i}$.

From the form of the solution (12) and (13), we can see that the computation of the updated eigenvalues and eigenvectors do not need to do eigen-decomposition all the time. Because of the approximate solution, there exists small loss of the system performance. In our forthcoming scheme, we applied the unused eigenvalue to be an indication of the channel quality, so the transmitter can do the power allocation according to it. The simulation has proved that it is a good scheme to compensate the performance loss.

\section{Proposed Power Allocation Scheme}

In the following, we propose a power allocation scheme which compensates the power loss due to approximated computation by perturbation theory. The SLNR precoding design in (3) also depends on the amount of power allocated to each user which takes the CSI into consideration. However in the practical wireless communication system, each user's channel fading is not completely the same. By (6), we can clearly see that the channel quality directly determine the SLNR at the receiver, it can significantly affect the system performance. Since the total transmission power of BS is constrained, if the transmit power is allocated according to the perfect CSI, we can get performance gains due to the improved powerefficiency.

The receivers feedback the liability CSI to the transmitter and the transmitter choose the preferable precoding vector to transmit the signal. We put forward a straight solution of a simplified power allocation problem. As the transmitter knows the perfect CSI, it allocates more power to the user which has better channel quality. The precoding vector is the eigenvector corresponding to the largest eigenvalue $\lambda$ of $\mathbf{H}_{k}^{\mathrm{H}} \mathbf{H}_{k}$, and it is in proportion to the channel power, so that the largest eigenvalue can be used as an indication to the quality of channel. Then, an SLNR-based power allocation scheme can be 
formulated as

$$
\frac{p_{k}}{p_{0}}=\frac{\lambda_{k}}{\sum_{i=1}^{\mathrm{K}} \lambda_{i}}
$$

The solution of the updated SLNR value $\lambda_{i}(\varepsilon)$ in (12) substituted in (14), the updated SLNR value is more accuracy to do the power allocation.

$$
\frac{p_{k}}{p_{0}}=\frac{\left(\lambda_{k}+\Delta \lambda_{k}(\varepsilon)\right)}{\sum_{i=1}^{\mathrm{K}}\left(\lambda_{i}+\Delta \lambda_{i}(\varepsilon)\right)}
$$

Using the updated SLNR to do power allocation, we can see the effect of parameters like eigenvalues, transmit power and channel perturbation on the capacity.

$$
\mathrm{C}_{p e r}=\sum_{k=1}^{\mathrm{K}} \log \left(1+\frac{\left(\lambda_{k}+\Delta \lambda_{k}(\varepsilon)\right) \mathrm{p}_{0}}{\sigma^{2} \sum_{i=1}^{\mathrm{K}}\left(\lambda_{i}+\Delta \lambda_{i}(\varepsilon)\right)}\right)
$$

When computing $\Delta \lambda_{k}$, we define $\mathbf{A}[\mathbf{n}]=\mathbf{H}_{k}^{\mathrm{H}}[\mathrm{n}] \mathbf{H}_{k}[\mathrm{n}]$ and $\mathbf{B}[\mathbf{n}]=\mathrm{M}_{k} \sigma_{k}^{2}+\mathbf{H}_{k}^{\mathrm{H}}[\mathrm{n}] \mathbf{H}_{k}[\mathrm{n}]$ at time $\mathrm{n}$.

In the computation of updated SLNR value, we also achieve performance gain benefits by adjusting the updated precoding vectors obtained from solution (13). The proposed simplified power scheme barely adds any additional calculation compared to the scheme only updating the precoding vectors and it has less computation amount compared to the conventional SLNR precoding scheme which do the eigen-composition every time step in the time-varying channel. So the proposed allocation scheme using updated eigenvalue can improve the system performance considering of less computation complexity.

\section{Numerical Results}

In this section, we evaluate the performance of the proposed algorithm. We consider a MU-MIMO system with $4(\mathrm{~K}=4)$ users. Assume the BS has $12(\mathrm{~N}=12)$ antennas, each user equipped with $2\left(\mathrm{M}_{k}=2\right)$ antennas and each user is transmitted with $2(\mathrm{~L}=2)$ streams. In this simulation experiment, the number of transmit antenna is more than the sum of all the receivers. All simulations are conducted using a QPSK modulation and a frame with 200 bits averaged over 10000 frames. We use the first order Gauss-Markov model to simulate a time-varying Rayleigh-fading channel. And the average bit error rate (BER) of users and system capacity are used as the evaluation criterion.

In Figure 2, we can clearly see that when we update the SLNR each time step, the loss of the SLNR can be notably reduced compared to the non-updated scheme. We simulate about 100 depth of time steps and it is obvious that calculating the updated SLNR with the perturbation theory, the SLNR loss is negligible. Figure 2 shows that the SLNR value degrades only 0.1 through the approximate calculation. However the non-update scheme degrades about 0.6 which demonstrates that the updated SLNR scheme will have small effects on the system performance with reduced computational complexity.

For the purpose of matching our hardware implemen in Figures $\mathbf{3}$ and $\mathbf{4}$, we compare the BER performance and system capacity of the proposed power allocation scheme using updated SLNR value (USLNR- PA) with MMSE algorithm (MMSE), the conventional SLNR maximization algorithm (CSLNR) and the updated precoding vectors scheme in [12] (SLNR-PERTUBATED). When the channel variation is not significant, the SLNR-PERTUBATED scheme only update the precoding vectors causes about $0.3 \mathrm{~dB}$ loss in BER and 0.2 $\mathrm{bps} / \mathrm{Hz}$ loss in capacity compared to the conventional SLNR precoding scheme. From the result, the proposed power allocation scheme shows about $0.5 \mathrm{bps} / \mathrm{Hz}$ additional capacity gain and $1 \mathrm{~dB}$ BER gain per user over the SLNR-PERTUBTED scheme. Although the average achievable BER and capacity of the proposed algorithm

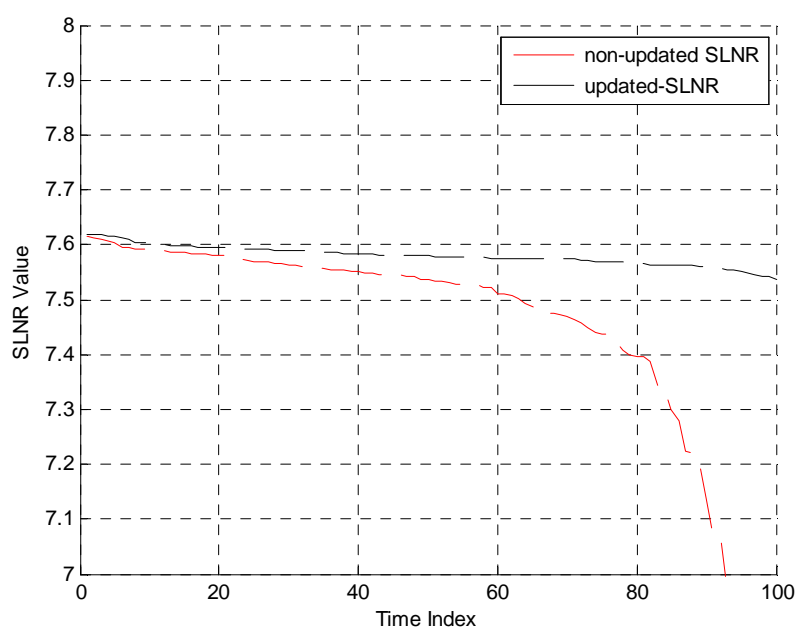

Figure 2. SLNR value degrade with time index.

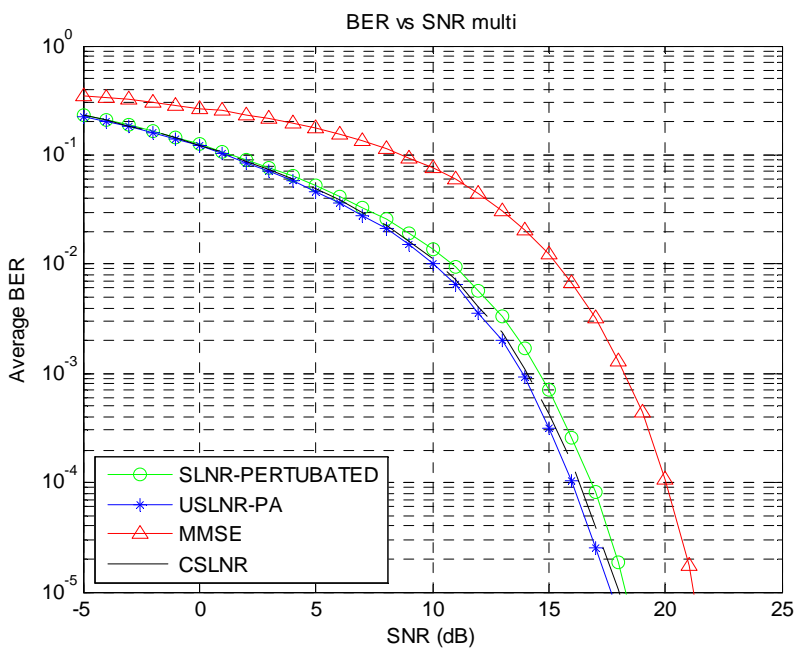

Figure 3. BER performance of $K=4, N=12, M_{k}=2$. 


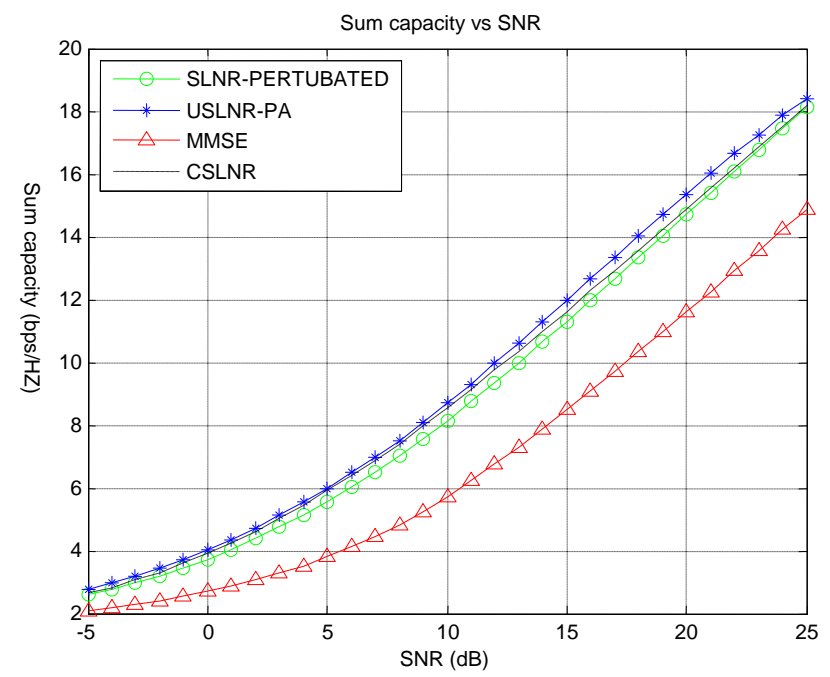

Figure 4. Sum capacity of $K=4, N=12, M_{k}=2$.

is small but the simplified power allocation scheme obtain the performance gain with no additional computation and it not only makes up the performance loss in [11], but also obtains the performance gain about $0.5 \mathrm{~dB}$ in BER and $0.3 \mathrm{bps} / \mathrm{HZ}$ in capacity compared with the conventional algorithm.

The results show that the proposed scheme has the best performance from both BER and system capacity. Using perturbation theory to obtain the updated SLNR and the updated precoding vector rather than decomposing the matrix to get the generalized eigenvalues and eigenvectors is an excellent way to achieve balance between algorithm complexity and system performance. What's more, using the updated SLNR value to do a power allocation can further improve the system performance. From (2), the SLNR precoding design depends on the amount of power allocated to each user so that allocating more power to the user having good channel quality can increase the system performance. This strategy to compensate the performance loss in [12] is feasible.

\section{Conclusions}

In this paper, we have investigated the power allocation scheme using the updated SLNR value base on perturbation theory. As the time-varying channel is taken into consideration, we avoid doing the eigen-decomposition in two consecutive time step. It leads to relatively less amount of calculation compared to the conventional SLNR algorithm and better system performance compared to the scheme only updating precoding vector. Then the proposed power allocation scheme using updated SLNR value which is more accuracy as an indicator to the channel quality compensates the performance loss caused by the approximate calculation.

\section{REFERENCES}

[1] C. Windpassinger, R. F. H. Fisher, T. Vencel, and J. B. Huber, "Precoding in multiantenna and multiuser communications," IEEE Trans. Wireless Communications, Vol. 3, No. 4, 2004, pp. 1305-1316. doi:10.1109/TWC.2004.830852

[2] M. Costa, "Writing on Dirty Paper," IEEE Transactions Information Theory, Vol. 29, No. 3, 1983, pp. 439-441. doi:10.1109/TIT.1983.1056659

[3] C. B. Ribeiro, K. Hugl, M. Lampinen and M. Kuusela, "Performance of Linear Multi-user MIMO Precoding in LTE System," Wireless Pervasive Computing, 2008. ISWPC 2008. 3rd International Symposium on, 7-9 May 2008, pp. 410-414.

[4] M. Joham, W. Utschick and J. A. Nossek, "Linear Transmit Processing in MIMO Communications Systems," IEEE Transactions Signal Process., Vol. 53, No. 8, 2005, pp. 2700-2712. doi:10.1109/TSP.2005.850331

[5] C. B. Peel, B. M. Hochwald and A. L. Swindlehurst, "A Vector-perturbation Technique for Near-capacity Multiantenna Multiuser Communication-Part I: Channel Inversion and Regularization," IEEE Transactions Communications, Vol. 53, No. 1, 2005, pp. 195-202. doi:10.1109/TCOMM.2004.840638

[6] Q. H. Spencer, A. L. Swindlehurst and M. Haardt, "Zero-forcing Methods for Downlink Spatial Multiplexing in Multiuser MIMO Channels," IEEE Transactions Signal. Processing, Vol. 52, No. 2, 2004, pp. 461-471. doi:10.1109/TSP.2003.821107

[7] Q. H. Spencer, A. L. Swindlehurst and M. Haardt, "Zero-Forcing Methods for Downlink Spatial Multiplexing in Multiuser MIMO channels,"

[8] M. S chubert and H . Boche, "Solution of the Multiuser Downlink Beam-forming Problem with Individual SINR Constraints," IEEE Transactions on Vehicular Technology, Vol. 53, No. 1, 2004, pp. 18-28.

[9] M. Sadek, A. Tarighat and A. H. Sayed, "A Leakage-based Precoding Scheme for Downlink Multi-user MIMO Channels," IEEE Transactions Wireless Communications, Vol. 6, No. 5, 2007, pp. 1711-1721. doi:10.1109/TWC.2007.360373

[10] A. Tarighat, M. Sadek and A. H. Sayed, "A Multi User Beam-forming Scheme for Downlink MIMO Channels Based on Maximizing Signal-to-leakage Ratios," in Proc. IEEE Int. Conf. Acoust., Speech, Signal Process., Philadelphia, PA, Mar. Vol. 3, 2005, pp. 1129 -1132.

[11] H. Yu, J. C. Shin and S.-k. Lee, "Multi-user MIMO Downlink Beamforming Based on Perturbation Theory of Generalized Eigenvector," Wireless Communication Systems (ISWCS), 2012 International Symposium on, pp. 141-145, 28-31 Aug. 2012.

[12] P. Viswanath, D. N. C. Tse and V. Anantharam, "Asymptotically Optimal Waterfilling in Multiple Antenna Multiple Access Channels," Information Theory, 2000. Proceedings. IEEE International Symposium on, pp. 466 , 2000.

[13] W. Yu, W. Rhee, J. M. Cioffi, "Optimal Power Control in Multiple Access Fading Channels with Multiple Anten- 
nas," Communications, 2001. ICC 2001. IEEE International Conference on, Vol. 2, 2001, pp. 575-579.

[14] H. Yu, Y. Sung, H. Kim and Y. H. Lee, "Beam Tracking for Interference Alignment in Slowly Fading MIMO Interference Channels: A Perturbations Approach Under a
Linear Framework," Signal Processing, IEEE Transactions on, Vol. 60, No. 4, 2012, pp. 1910-1926.

[15] R.-C. Li, "Matrix Perturbation Theory," http://www.uta.edu/faculty/rcli/papers/li2007HLA.pdf. 